\title{
Design and Implementation of Information Management and Decision System by Mobile Intelligent Terminal in Bridge Inspection
}

\author{
Xiaochuan $\mathrm{He}^{1}$, Jinghua $\mathrm{Fu}^{*}{ }^{2}$ a , Tian Xia ${ }^{1, \mathrm{~b}}$, Xiao Zhou ${ }^{1}$ and Li Ding ${ }^{3}$ \\ ${ }^{1}$ Wuhan Roads \& Bridges Toll Management Center, Wuhan, China \\ ${ }^{2}$ National Engineering Laboratory for Fiber Optic Sensing Technology, Wuhan Univ. of Technology, \\ Wuhan, China \\ ${ }^{3}$ Wuhan WUTOS Co.,Ltd, Wuhan, China \\ a foryoufirst@163.com, ${ }^{\text {b } 475866149 @ q q . c o m ~}$
}

\begin{abstract}
Keywords: artificial inspection, mobile intelligent termination, bridge management, maintenance Abstract. The information management and decision system of artificial bridge inspection is based on android mobile intelligent terminal which can make artificial and regular inspection electronic, information and networking, and improve the working efficiency and the level of bridge management and maintenance.
\end{abstract}

\section{Introduction}

The research of bridge management system in China since the 1980s, on the basis of abroad experience, provinces of Sichuan, Guangdong, Henan and Beijing had developed their own distinctive bridge management system. However, these systems are still in the experimental stage. The establishment of the model parameters needs to analyze the accumulation of a large number of historical data, and the level of the system itself is also not clear. Therefore, these systems are still needs further development and improvement.

Relative large number of bridges under the jurisdiction of a limited number of professional bridge inspection personnel and lack of information methods mean that the current bridge structure security management in the following areas still lacking:

(1) Detection and evaluation results of each bridge is isolated paper report and the test results do not form interactive electronic information sharing which are not conducive to the development of efficient information between transverse and longitudinal association, comparison, statistics and trend analysis.

(2) Bridge maintenance and daily safety management emphasis on traditional manual methods which do not form a digitalization, information, networking and visualization office mode.

\section{Software system design}

Artificial bridge inspection information acquisition subsystem is installed on a handheld Android tablet computer. Inspection personnel fill out the electronic maintenance manual instead of traditional paper documents. After the inspection is completed, the handheld tablet computer accesses the LAN of monitoring system to transmit digital inspection data. The decision-making subsystem for the management of artificial inspection data can be deployed in the information center. Inspectors through the system to make the data upload to the bridge management center server.

Based on detailed analysis of the existing bridge maintenance and management contents, we identified the following two subsystems of software development: artificial bridge inspection information acquisition subsystem by handheld mobile intelligent terminal, the decision-making and management subsystem of inspection data. The first one is responsibility for assisting users to complete standardization of the inspection information. The other one is mainly responsible for receiving artificial inspection data to the subsystem and completion of data storage, statistics, query management, a detailed analysis of the evaluation, output and print corresponding statement etc. System-level association shown in Figure 1. 


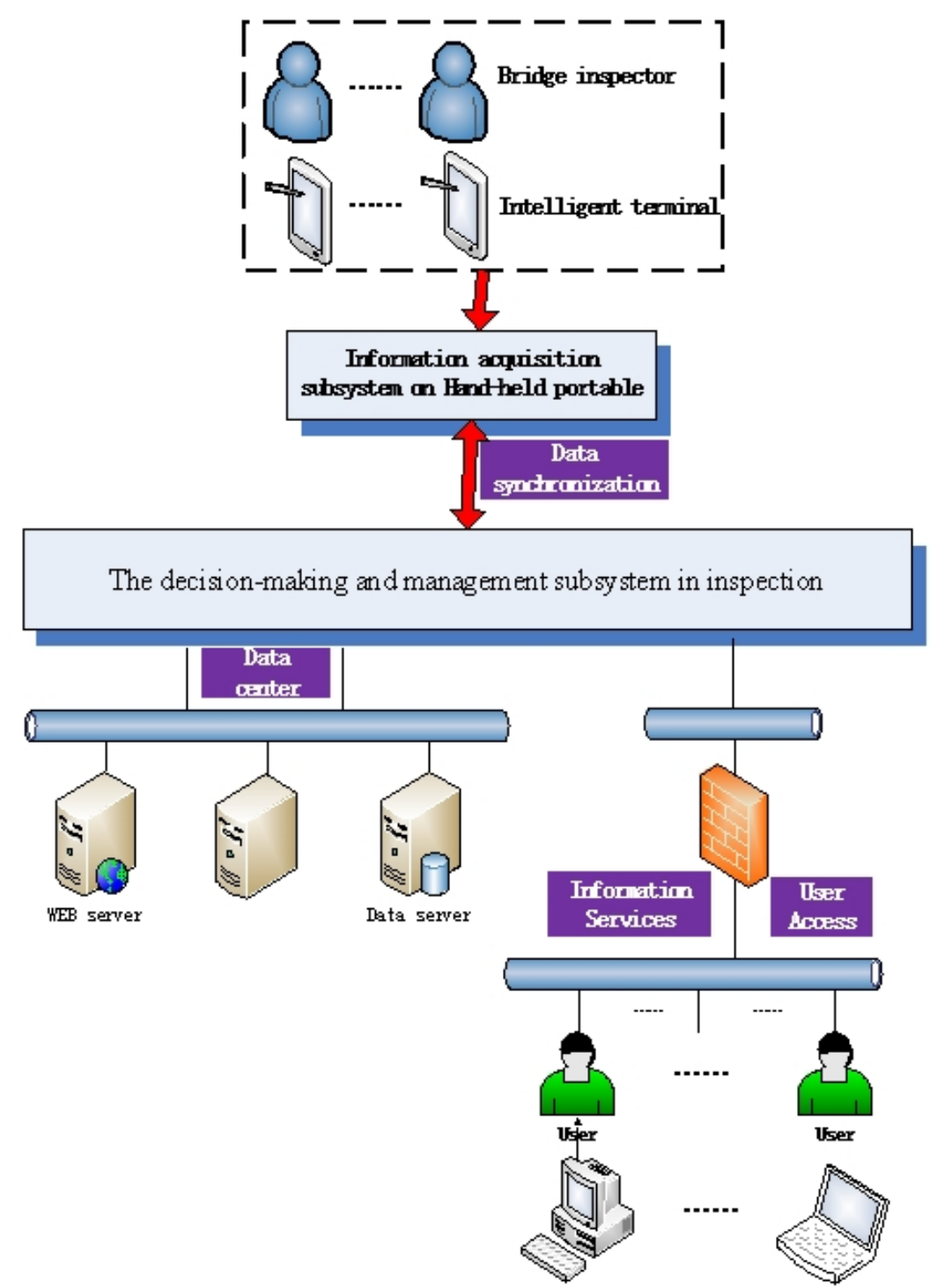

Figure1 association diagram for the decision-making and management subsystem of inspection The main function of mobile intelligent terminal subsystem:
(1) On-site inspection information input
(2) Query information
(3) Modify and edit
(4) Data upload

The management and decision subsystem of inspection information adopts $\mathrm{B} / \mathrm{S}$ structure. The main features include:

(1) Inspection data management

(2) Statistics and inquiries

(3) Report generation

(3) Analysis and evaluation

\section{Bridge inspection classification}

The system for Wuhan Yangtze River Second Bridge is customization. According to the work mode of the maintenance department of this bridge, there are three parts in inspection inputting, included daily inspection, regular inspection and outsourcing inspection:

(1)Daily inspection: the inspection personnel will do this on a daily basis, mainly for visual inspection of the facilities for the deck, superstructure, substructure and subsidiary structures. This bridge specially increases power supply and distribution system and ETC charging facilities inspection records in daily inspection. 
(2)Regular inspection: according to the characteristics of bridge structural members, the inspection frequency may be once every three months, every six months or once every year. The process of inspection needs the help of related professional testing instruments. From the result and specification, there are scores for bridge component and the whole bridge in regular inspection.

(3)Outsourcing inspection: specialized unit will have an annually detection for full-bridge cable force and its linear.

\section{The design of bridge daily inspection system}

Daily inspection system is mainly for inspection personnel to use. Its function is to make the inspection process standardization and the inspection information electronic and so on. The maintenance manual of Wuhan Yangtze River Second Bridge and the current inspection reports can determine the content and process of daily inspection. While it adds additional features to record attendance of inspection personnel and draw the inspection route through the two-dimensional code scanning.

Handheld mobile intelligent terminal information acquisition subsystem of bridge daily inspection complies with the following principles:

1) The 7 inch Android system PAD as the mobile intelligent terminal.

2) Taking account of its characteristic, PAD only input information. The other functions such as analysis and statistics are on the WEB side.

3) All the possible defects of bridge member in all kinds of inspection are input to the system beforehand. Operators only need to choose options on PAD, no need for text entry.

4) Only records the defect.

The software screenshots of daily inspection information acquisition subsystem on PAD are shown in figure 2.
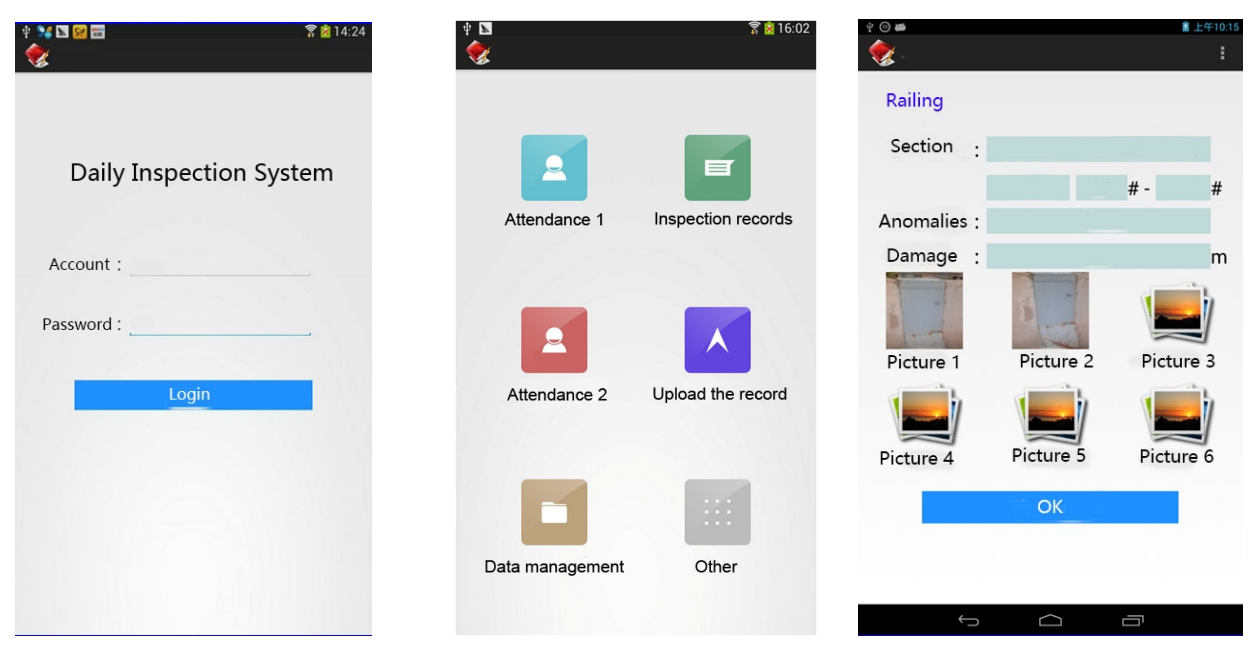

Figure2 software screenshot of daily inspection on PAD

The decision-making and management subsystem of daily inspection or WEB can afford some functions, such as inspection results management, inspection calendar and inspection results generation and inquiry. Some screenshots of this subsystem is shown in figure 3. 

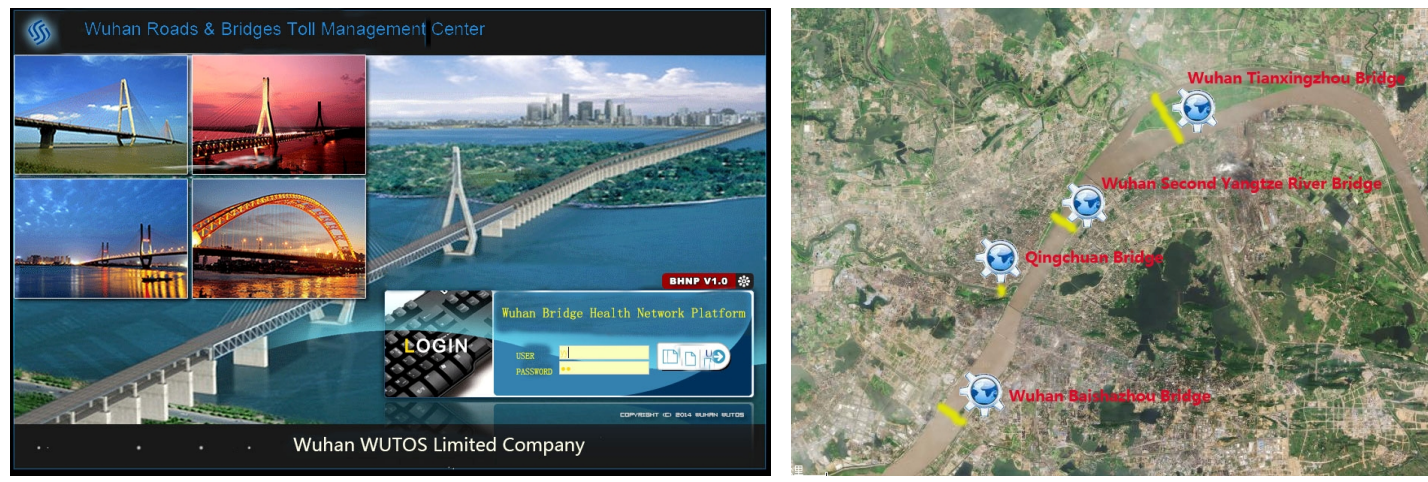

Figure 3 Screenshots of WEB software

\section{The design of bridge regular inspection system}

The inspection personnel who use the regular inspection are more professional than the daily inspection personnel, most of them are professional bridge maintenance personnel. Regular inspection system is more complex than the daily inspection, after the regular inspection, we need to grade bridge members according to the standards for technical condition evaluation of highway bridges .

The core of regular inspection system is to describe bridge member defects with accurate correlation of scale in the specification.

The regular inspection system complies with the following principle:

(1) According to the maintenance specification in regular inspection, making the 18 forms electronic. And there is the function of remind with the inspection tools.

(2) The defect standard of bridge member can establish evaluation database. Only inputting the value of defects, the system can automatically determine the defect level.

Screenshots of software below:
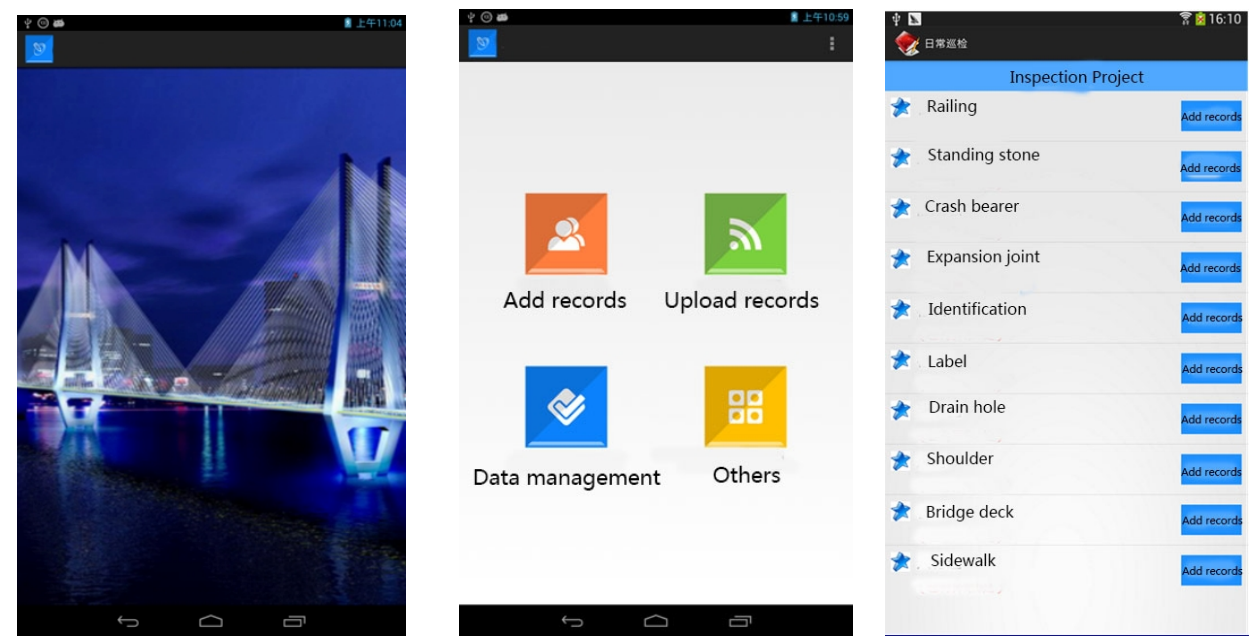

Figure4 screenshots of regular inspection software on PAD

Regular inspection system in the WEB has the same function with daily inspection system, such as inspection results management, inspection calendar and inspection results generation and inquiry. Regular inspection date inputting function is added in the system.

\section{Conclusion}

Currently, the management and decision system of artificial bridge inspection based on mobile intelligent terminal had been used in the management and maintenance work of Wuhan Yangtze River Second Bridge and gradually made the management and maintenance work electronic, information and networking. 


\section{Acknowledgements}

This work was financially supported by the Fundamental Research Fund for the Central Universities(WUT:2014-IV-009, WUT: 2014-II-012).

\section{References}

[1] Shen wang, Zhang qiang, in: Electronic bridge inspection and maintenance management, Engineering Sciences. 57-60(2010).

[2] Ministry of Transport of the People's Republic of China: Standards for technical condition evaluation of highway bridges edited by China Communications Press, Beijing, 2004.

[3] Technical code of maintenance for city bridge (CJJ99-2003) [S]. Beijing: China Architecture \& Building Press, 2004.

[4] The Bridge Toll Management Center of Wuhan, china railway major bridge reconnaissance \& design institute co, ltd: Bridge maintenance and management manual -Wuhan Yangtze River Second Bridge Technical Volume (Wuhan, 2012). 